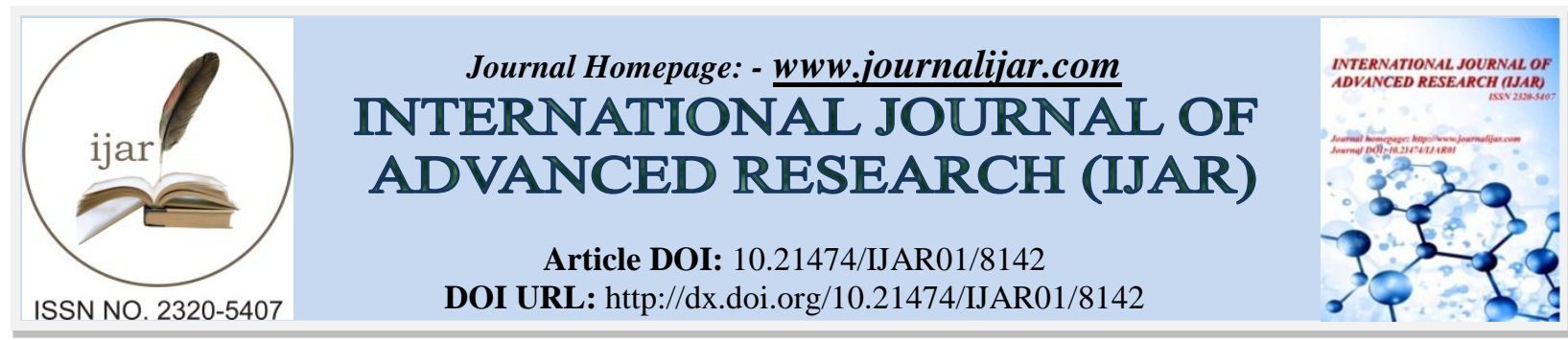

RESEARCH ARTICLE

\title{
POSSIBILITY OF MEASUREMENT OF X-RAY OUTPUT FROM A MAMMOGRAPHIC X-RAY APPARATUS BY USE OF SCATTERED RADIATION.
}

\author{
Honami Akiba $^{1}$, Michiharu Sekimoto ${ }^{2}$, Yoh Katoh ${ }^{3}$, Michiko Nemoto $^{4}$, Kohei Nishi ${ }^{5}$ and Akio Okumura ${ }^{6}$. \\ 1. Radiology Department, Tokyo Metropolitan Cancer and Infectious Diseases Center Komagome Hospital. \\ 2. Faculty of Medical Technology, Niigata University of Health and Welfare. \\ 3. Faculty of Health Sciences, Tokyo Metropolitan University. \\ 4. Office of Radiation Technology, Keio University Hospital. \\ 5. JR Tokyo General Hospital. \\ 6. Radiology Department, Tokyo Metropolitan Cancer and Infectious Diseases Center Komagome Hospital.
}

\section{Manuscript Info}

Manuscript History

Received: 01 October 2018

Final Accepted: 03 November 2018

Published: December 2018

\section{Keywords:}

mammography, AGD, semiconductor

dosimeter, scattered radiation, added filter.

\section{Abstract}

In June 2015, Diagnostic Reference Levels was drawn up in Japan. Therefore, the exposure control of the examinees is more important than ever. In this research, in order to grasp the AGD for each examinee, a basic examination of a method to measure $\mathrm{X}$ - ray output without changing the quality of the beam was made. In order not to change the quality of the radiation, scattered radiation from the added filter is used and the added filter is mounted in the X-ray source device, so it is necessary to dispose the dosimeter also in the X-ray source device. It was measured with a semiconductor dosimeter.

The measurement conditions, that are likely optimal judging from the examination results, are as follows: (1) The semiconductor dosimeter is to be cooled constantly due to its temperature characteristics. (2) The straight line connecting the added filter center and the semiconductor dosimeter's detector center is to be orthogonal to the detection plane of the dosimeter's detector constantly. (3) The dosimeter's detector is to be opposed, at an angle not blocking the primary X-ray, to the added filter as much as possible.

It is likely possible to calculate the X-ray output in mammographic Xray apparatuses by multiplying the dose of the scattered radiation measured in the layout above by the respective calibration constant of each target/added filter combination. In addition, we believe that it is possible to grasp AGD by examinee by multiplying the X-ray output by coefficients additionally.

Copy Right, IJAR, 2018,. All rights reserved.

\section{Introduction:-}

In June 2015, Japan Network for Research and Information on Medical Exposures (J-RIME) drew up Diagnostic Reference Levels (DRLs) in Japan. These DRLs 2015 are by no means the upper limits of radiation dose, but only the means for management of dose received by patients so that balance between diagnostic and therapeutic purposes is kept. As for the DRL in mammography in the above, a value of " $2.4 \mathrm{mGy}$ as average glandular dose (AGD)" was presented. Mammography generally produces a low standard deviation indicating a compact dose distribution as 
compared to other modalities. This appears attributable to accuracy management that has been conducted by a number of academic societies including the Japan Central Organization on Quality Assurance of Breast Cancer Screening, a nonprofit organization (NPO). In addition, data accumulation uses mammographic X-ray apparatuses that are A/B-certified based on checking of images taken in facilities, and is also likely to involve intervention by many accredited technologists. For that reason, 95 percentile is applied to the DRL setting.

A fact that tissue weighting factor of the mammary gland is high has begun spreading an approach of using AGD, the dose absorbed at the mammary gland position, in mammographic risk assessment. Because it is difficult to measure the absorbed dose directly, it is general to find AGD by multiplying incident air kerma (IAK) at a breast surface position by a coefficient given by polymethyl methacrylate (PMMA) thickness and half-value layer (HVL), and a coefficient related with target/added filter combination. HVL and IAK are measured by use of a plane-parallel ionization chamber dosimeter. According to the guidelines currently adopted in Japan, AGD in the breast with 50\% mammary gland content, obtained eventually through multiplication by various coefficients, is used.

However, actual AGD differs because percentage of mammary gland content and/or breast thickness vary depending patient. It is impossible to calculate actual AGD in individual patients while they are under mammography because use of this calculation method disturbs the photography due to the presence of the dosimeter. If an area dosimeter is used as in interventional radiology (IVR), the dose can be measured without disturbing the photography. However, attachment of an area dosimeter to the irradiation port leads to beam hardening. Because mammography uses an Xray of very low energy for improvement of contrast, an influence of beam hardening appears on images, which is likely to influence diagnosis. Therefore, use of an area dosimeter is unsuitable for mammography.

Taking into consideration the background above, this study aimed to examine a method of measuring X-ray output without changing radiation quality, for grasping exposure dose by examinee.

\section{Materials and Methods:-}

Currently, many mammographs display AGD on their console screens after every irradiation. However, displayed AGD is not a value measured in the examination, but an estimated value calculated from target/added filter, tube voltage, breast thickness, etc. chosen in pre-irradiation. Because of the presence of the breast at the position of dosimeter in actual AGD measurement in each examination, currently no apparatus that can measure actual AGD of individual examinees is available, so that exposure is reduced by quality control and quality assurance using reference phantoms. Therefore, this study proposed a method of measuring actual AGD of individual examinees. In order not to change radiation quality used in the photography, we planned out using scattered radiation generated by use of an added filter instead of direct radiation. As figure 1 shows, each added filter is very small measuring $4 \mathrm{~cm} x$ $5 \mathrm{~cm}$ approximately, and arranged in an X-ray source system. For that reason, we determined to use a semiconductor dosimeter which has high sensitivity and a small detector which can be arranged in an X-ray source system. Taking these into consideration, following five items were examined.

1. Temperature characteristics of the semiconductor dosimeter

2. Incidence direction characteristics of the semiconductor dosimeter

3. Angle characteristics of added filters and the detector of the dosimeter

4. Spectrum of scattered radiations from added filters

5. Relationship between scattered radiation dose and dose of radiation transmitted through an added filter

In the semiconductor dosimeter used in this study, which is composed of Solidose 308 (RTI Co.) as detector and Solidose 308 electrometer as monitor, the material uses $\mathrm{Si}$, and a depletion layer of a p-n junction acts as sensitive layer to radiation. The X-ray source system used in the measurements is composed of an industrial X-ray generator ISOVOLT Titan E (GE Co.), and X-ray tubes ISOVOLT 160 (W target) and FA100/3 (Mo target). This system allows us to manually switch target between $\mathrm{W}$ and Mo, highly stably outputs X-ray, and allows us to attach an additional filter arbitrarily. The target/added filter combinations were W/0.5-mm Al, W/0.02-mm Ag, W/0.025$\mathrm{mm} \mathrm{Rh}, \mathrm{Mo} / 0.03-\mathrm{mm}$ Mo, and Mo/0.025-mm Rh due to limitation in added filters we possessed.

\section{Temperature characteristics of the semiconductor dosimeter:-}

Electric resistance of metals becomes higher with temperature rise in near proportion to absolute temperature. However, in the case of semiconductors, generally the resistance decreases suddenly as temperature rises. In addition, change of electric resistance of semiconductors by temperature is considerably drastic. Their absolute values of temperature coefficient are higher than those of metals by one digit or more ${ }^{4)}$. Thus, semiconductors are 
influenced by ambient temperature, so that measurement values by the semiconductor dosimeter are likely to be influenced by temperature. Therefore, temperature characteristics of the semiconductor dosimeter were examined.

Figure. 2 shows the layout in the measurement regarding temperature characteristics. The semiconductor dosimeter and a thermometer were placed in the inside, which was surrounded by a sheet-like heater, of a self-fabricated Styrofoam container. The dosimeter was arranged so that its reference plane would be vertical to the X-ray beam, and temperature was increased from $22.8^{\circ} \mathrm{C}$ up to $60^{\circ} \mathrm{C}$ in the measurement. The distance between the focal point and the reference plane of the dosimeter was $650 \mathrm{~mm}$, the lower limit with which the semiconductor dosimeter could come close to the focal point. The measurement conditions were: tube voltage, $30 \mathrm{kV}$; tube current, $5 \mathrm{~mA}$; and irradiation duration, $10 \mathrm{~s}$. The target/added filter was W/Al.

Incidence direction characteristics of the semiconductor dosimeter:-

Semiconductor dosimeters are dependent on X-ray incidence direction though they have good sensitivity ${ }^{3)}$. Therefore, an incidence direction with which measurement values could be obtained most efficiently was examined.

Figure. 3 shows the layout drawing of the measurement regarding incidence direction characteristics. The distance between the focal point and the reference plane of the dosimeter was $1000 \mathrm{~mm}$, the lower limit with which the semiconductor dosimeter could come close to the focal point. In the measurement, the reference plane of the dosimeter was rotated from $0^{\circ}$ to $180^{\circ}$ in steps of $10^{\circ}$, in which $90^{\circ}$ was the angle with which the plane would be vertical to the X-ray beam. The measurement conditions were as follows.

Angle characteristics of added filters and the detector of the dosimeter:-

Scattered radiation from added filters is so weak that the dosimeter needs to be arranged at a position where the highest measurement value can be obtained. Therefore, angle characteristics of added filters and the detector of the dosimeter were examined.

Figure. 4 shows the layout in the measurement regarding angle characteristics of added filters and the detector of the dosimeter. The distance between the focal point and the reference plane of the dosimeter was $1000 \mathrm{~mm}$, the lower limit with which the semiconductor dosimeter could come close to the focal point. Each added filter was rotated around its center axis from $0^{\circ}$ to $30^{\circ}$ in steps of $10^{\circ}$ (four patterns). This filter angle was to be up to $30^{\circ}$ considering the angle which was likely to be actually arranged in mammographic X-ray apparatuses, by referring to about $28^{\circ}$ of the angle of the filter shown in figure. 1. The semiconductor dosimeter was arranged so that the distance between the center of the reference plane of the dosimeter and the center of each added filter would be 100 mm constantly (lower limit distance with which the dosimeter could come close to the filter), and rotated from $0^{\circ}$ to $60^{\circ}$ in steps of $10^{\circ}$ (seven patterns). All possible combinations of angles of each added filter and the semiconductor dosimeter were subjected to the measurement.

\section{Spectrum of scattered radiations from added filters:-}

The measurement by use of scattered radiation is on condition that scattered radiation quality is constant for tube voltage and measurement angle. Therefore, radiation quality by combination of target/added filter and tube voltage was examined.

Figure. 5 shows the layout drawing of the measurement. The distance between the focal point and the reference plane of the dosimeter was $1000 \mathrm{~mm}$ whereas the distance between the added filter center and the CdZnTe semiconductor detector was $130 \mathrm{~mm}$ (lower limit distance with which each added filter could come close to the semiconductor detector). X-ray spectrum was detected by use of a CdZnTe semiconductor detector RAMTEC415 (Toyo Medic). Each added filter was fixed at $0^{\circ}$ whereas the $\mathrm{CdZnTe}$ semiconductor detector was rotated from $20^{\circ}$ to $60^{\circ}$ in steps of $10^{\circ}$ to perform the measurement in the following photography conditions.

\section{Relationship between scattered radiation dose and dose of radiation transmitted through an added filter:-}

The presence of correlation between dose of scattered radiation generated by an added filter and dose of radiation transmitted through the added filter may allow us to calculate ESD of the breast from the scattered radiation dose. Therefore, scattered radiation doses from added filters and doses of radiations transmitted through the added filters were measured to examine their relationship. 
Figure. 6 shows the layout drawing of the measurement. The distance between the focal point and the reference plane of the dosimeter was $1000 \mathrm{~mm}$ whereas the distance between the added filter center and a free air chamber $(\text { FAC })^{5)}$ was $200 \mathrm{~mm}$. Each added filter was fixed at $0^{\circ}$, whereas the semiconductor dosimeter was at the position of $60^{\circ}$ where scattered radiation dose was likely to be detectable most abundantly. Scattered doses from the added filters were measured by the semiconductor dosimeter, whereas doses of radiations transmitted through the added filters were measured by the FAC.

\section{Results and Discussions:-}

Temperature characteristics of the semiconductor dosimeter:-

Figure. 7 shows measurement results regarding temperature characteristics. The measurement value at each temperature is represented by a relative value to the measurement value at $22.8^{\circ} \mathrm{C}$, room temperature. When the temperature in the Styrofoam exceeded about $55^{\circ}$, a value due to leakage current, which was $2 \mathrm{mGy} / 10 \mathrm{~s}$, was displayed.

This current flowing in a condition in which no X-ray is irradiated and only biased voltage is charged becomes a factor lowering $\mathrm{S} / \mathrm{N}^{6}$. When temperature exceeds $55^{\circ} \mathrm{C}$, a leakage current is generated, which may affect measurement values. The actual temperature inside the X-ray source system, which cannot be measured, is expected to become very high. Therefore, circumference of the semiconductor dosimeter should be cooled constantly for its use. Use of a Peltier element for the cooling is on our plan. It is likely possible to arrange a Peltier element in conjunction with the detector of the semiconductor dosimeter in the X-ray source system.

\section{Incidence direction characteristics of the semiconductor dosimeter:-}

Figure. 8 shows measurement results regarding incidence direction characteristics. Data are represented by relative values to the maximum measurement value. In the presence of a Mo target, it was in a case where the reference plane of the dosimeter was vertical $\left(90^{\circ}\right)$ to the X-ray beam that the highest measurement value was obtained when the tube voltage was $26 \mathrm{kV}$. In this condition, the $90^{\circ}$ arrangement led to the maximum value even when the tube voltage was changed. Because the same characteristics were seen even if tube voltage was changed, the measurement in the presence of a W target used only $30 \mathrm{kV}$. In this case, the $90^{\circ}$ arrangement led to the maximum value as in the above.

It was revealed that measurement values of the semiconductor dosimeter are barely dependent on neither tube voltage nor target, and the maximum value can be obtained at $90^{\circ}$. In addition, the used semiconductor dosimeter is likely to be able to measure only primary scattered radiations because the dosimeter, which has above $95 \%$ of relative sensitivity as long as its reference plane is positioned within $\pm 5^{\circ}$ from its orthogonal direction, becomes less sensitive abruptly at a higher angle. Therefore, it is likely possible to measure primary scattered radiations efficiently by arranging the reference plane of the detector of the semiconductor dosimeter so that the plane will be vertical to the straight line connecting the added filter center and the dosimeter's detector center.

\section{Angle characteristics of added filters and the detector of the dosimeter:-}

Figures. 9-13 show angle characteristics of added filters and the detector of the dosimeter. Data are represented by relative values to the maximum measurement value. The angle of the dosimeter's detector was to be up to $60^{\circ}$ because higher than $60^{\circ}$ would disable the measurement of a scattered radiation due to blockade of the primary Xray. The values when the dosimeter's detector was positioned at $0^{\circ}$ and $10^{\circ}$ were too low to measure. The relative measurement values became close to 1 as the angle of the semiconductor dosimeter became higher, and as the angle of each added filter became higher, in all target/added filter combinations. Any differences that were caused by a difference in tube voltage were not seen. When the added filter in Mo/Mo as well as W/Ag was positioned at $30^{\circ}$, nearly the same dose was obtained regardless of the angle of the dosimeter's detector. From the above, it is likely that a scattered radiation has a homogeneous distribution in all directions from the added filter center. In cases of $\mathrm{Mo} / \mathrm{Rh}, \mathrm{W} / \mathrm{Rh}$, and W/Al, measurement values are changed depending upon the angle of the dosimeter's detector when the added filter was positioned at $30^{\circ}$. Therefore, generation of a scattered radiation by $\mathrm{Rh}$ and $\mathrm{Al}$ filters is likely to have an angular distribution.

It is likely because of the following that the maximum value is obtained: the angle of the semiconductor dosimeter to an added filter becomes close to $90^{\circ}$ as the angels of both the dosimeter and the filter become higher. Scattered radiations, that are very weak originally, should be measured at a position where the maximum value can be 
obtained. For that reason, it is likely necessary to arrange the semiconductor dosimeter in a position not blocking the primary X-ray and in a way in which the angle formed with the added filter and the dosimeter's detector centers will be nearly perpendicular $\left(90^{\circ}\right)$ where possible.

\section{Spectrum of scattered radiations from added filters:-}

Figures. 14-18 show spectrograms linked to target/added filter combinations. Data are represented by relative values to the maximum measurement value. An X-ray characteristic to the material of an added filter, as well as a $\mathrm{K}$ absorption edge were observed. At a same tube voltage, any differences in the spectrum, that were caused by a difference in the angle of the CdZnTe detector to the added filter, were not seen.

As above, any combinations of angles of an added filter and the semiconductor dosimeter's detector did not change the radiation quality. Hence, no problem is likely to occur whichever angle the measurement is done with.

\section{Relationship between scattered radiation dose and dose of radiation transmitted through an added filter:-}

Figures. 19-23 show measurement results regarding relationship between scattered radiation dose and dose of radiation transmitted through an added filter. Each result was subjected to linear approximation to calculate the coefficient of determination. The values of coefficient of determination, $\mathrm{R}^{2}$, are in a range from 0.99649 to 1 , allowing us to say that proportionality is seen. A proportionality is nearly seen between measurement values of scattered radiation dose and dose of radiation transmitted through an added filter at all target/filter combinations. Meanwhile the inclination differed by tube voltage in $\mathrm{Mo} / \mathrm{Mo}, \mathrm{Mo} / \mathrm{Rh}, \mathrm{W} / \mathrm{Rh}$, and W/Ag combinations, the inclination in W/Al was the same even if tube voltage varied. Mainly $\mathrm{Si}$ is used for the detection medium in semiconductor dosimeters, in which a depletion layer of a p-n junction acts as sensitive layer to radiation ${ }^{7)}$. Because the surface $\mathrm{SiO}_{2}$ layer and the dead layer absorb X-ray highly, response of semiconductor dosimeters is strongly dependent on energy in case of a low-energy range of X-ray. In cases of Mo, Rh, and Ag filters, a characteristic Xray is emitted (Figures. 19-22). It is likely because of this characteristic X-ray that the inclination varied in use of Mo, Rh, and Ag filters.

It is likely possible to calculate the dose of radiation transmitted through an added filter by multiplying the scattered radiation dose by the inclination in this graph as calibration constant. ESD is found by applying the inverse square law of the distance to the breast surface to this dose of the radiation transmitted through the added filter. It is likely possible to calculate AGD by additionally multiplying this by various coefficients ${ }^{8)}$.

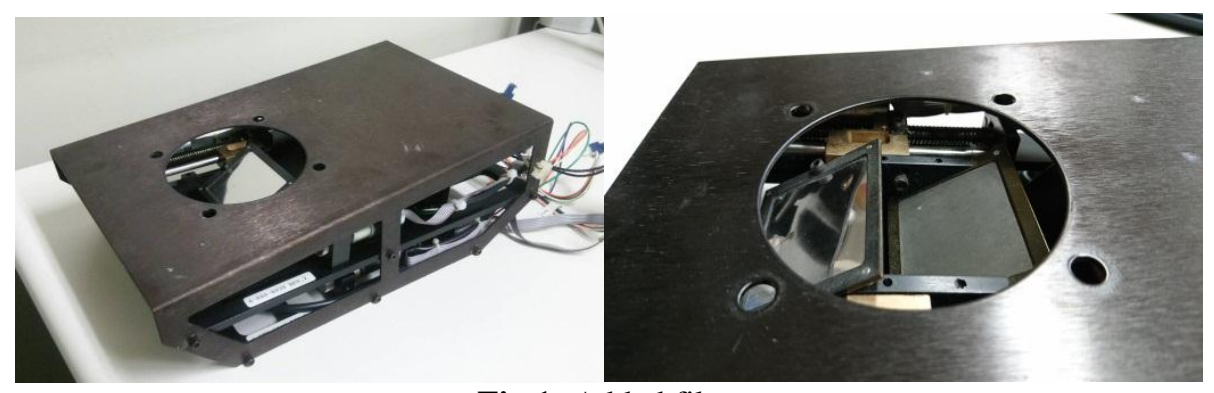

Fig 1:-Added filter 


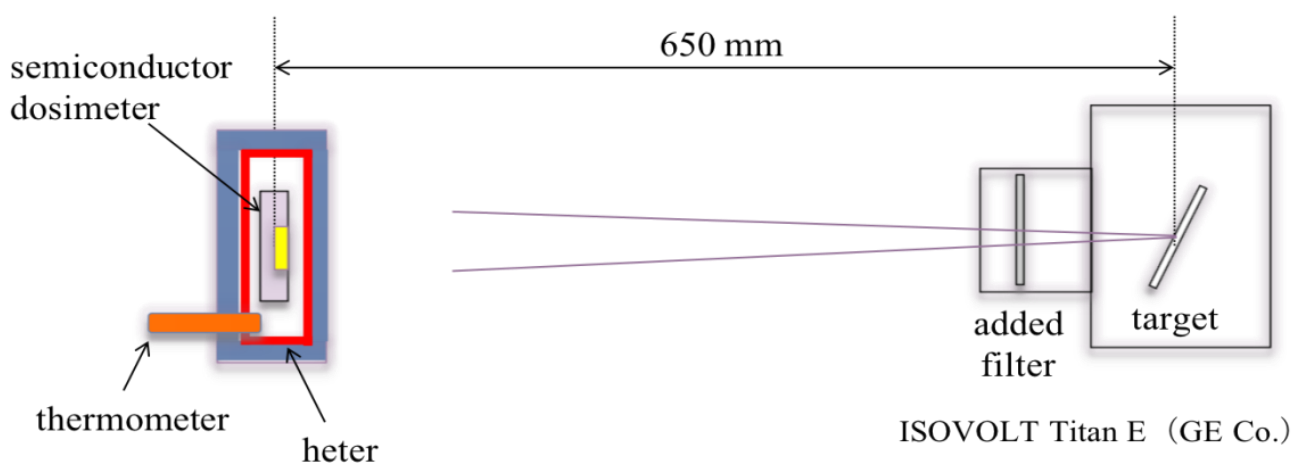

Fig 2:-The layout in the measurement regarding temperature characteristics

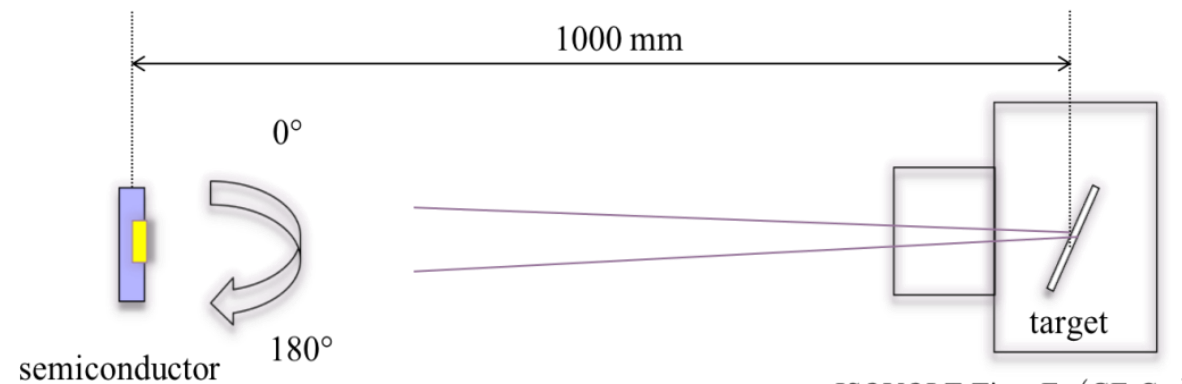
dosimeter

ISOVOLT Titan E (GE Co.)

Fig 3:-The layout of the measurement regarding incidence direction characteristics

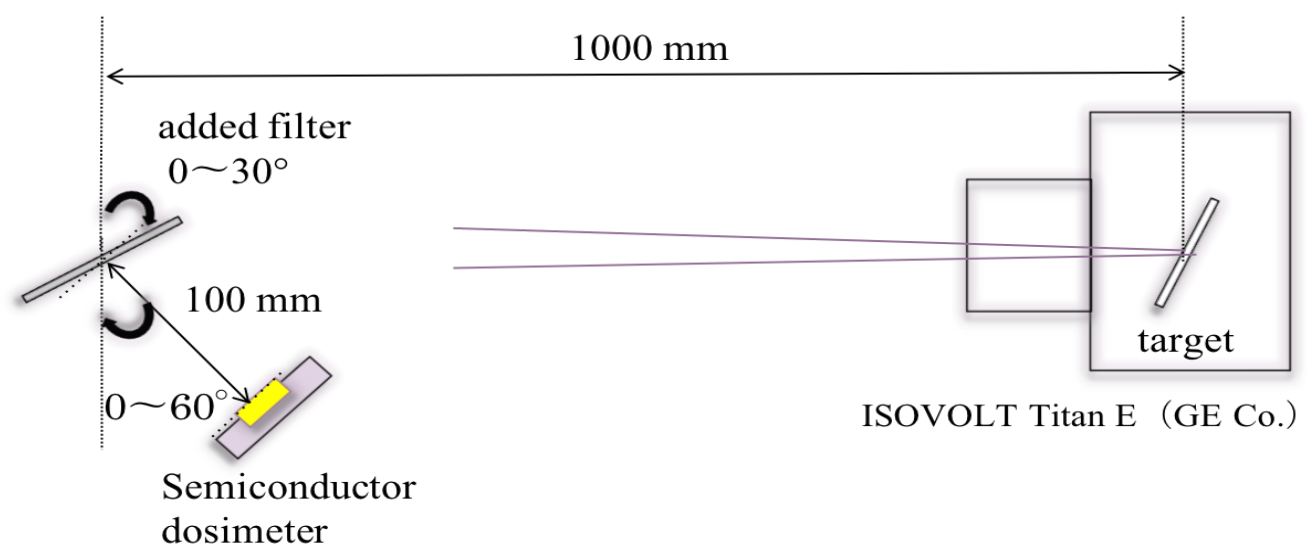

Fig 4:-The layout in the measurement regarding angle characteristics of added filters and the detector of the dosimeter 


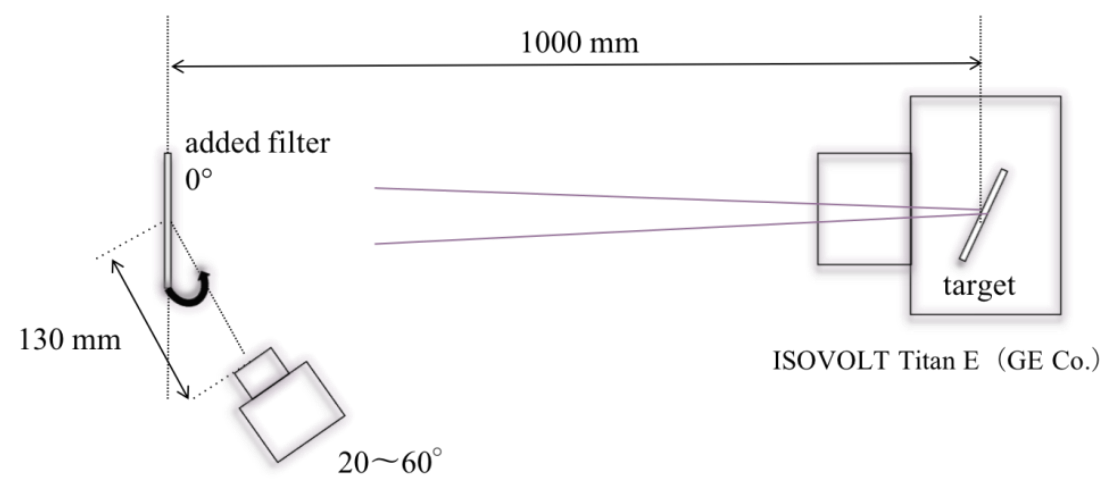

$\mathrm{CdZnTe}$ detector

RAMTEC415

(TOYO MEDIC Co.)

Fig 5:-The layout of the measurement regarding spectrum of scattered radiations from added filters

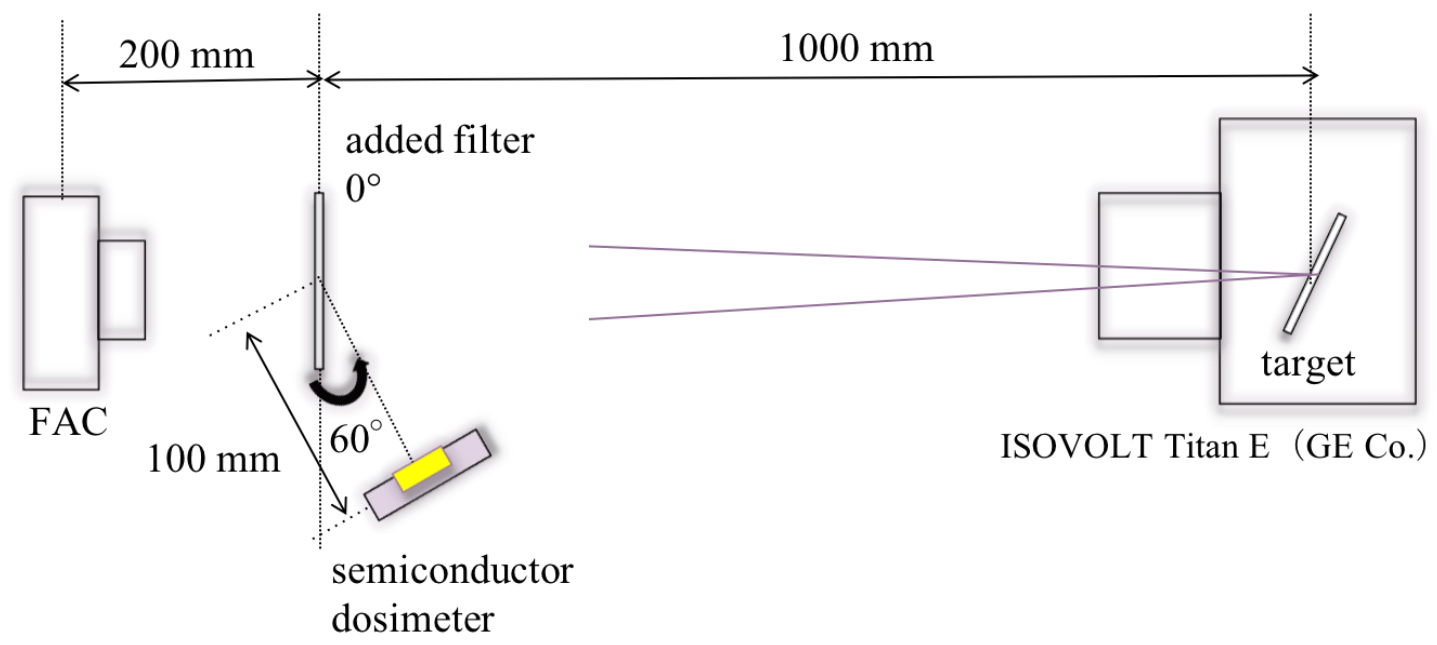

Fig 6:-The layout of the measurement regarding relationship between scattered radiation dose and dose of radiation transmitted through an added filter

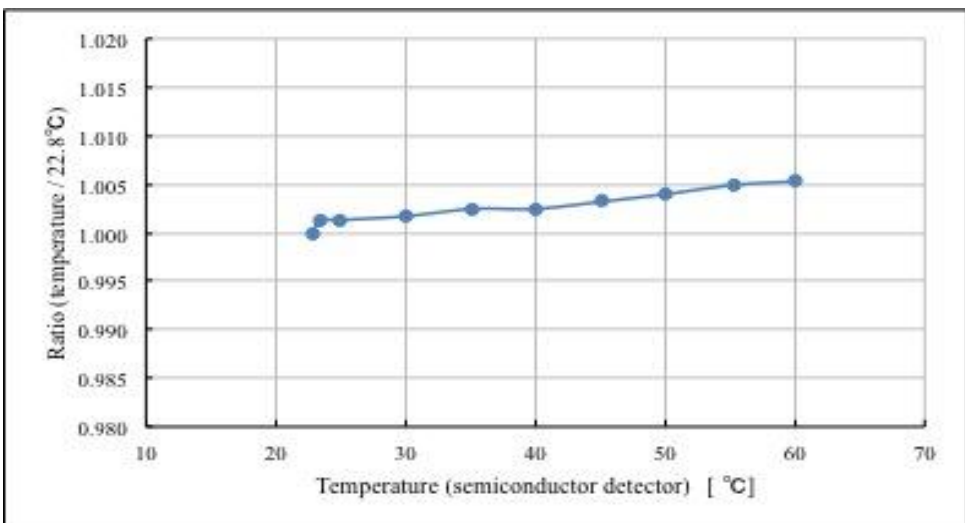

Fig 7:-Measurement results regarding temperature characteristics 


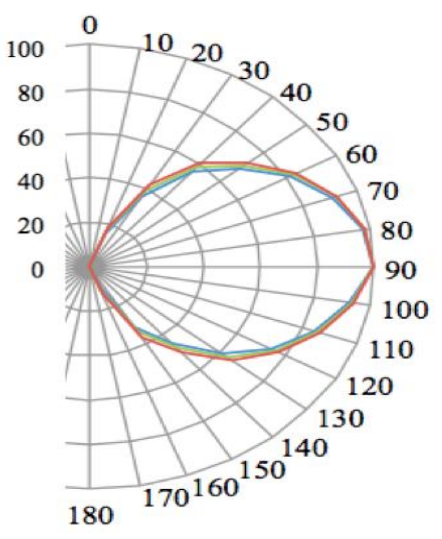

$\left[{ }^{\circ}\right]$

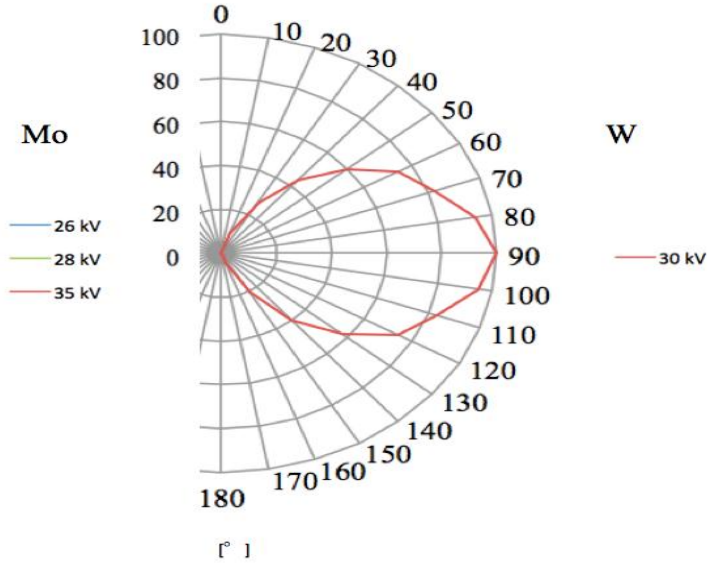

Fig 8:-Measurement results regarding incidence direction characteristics
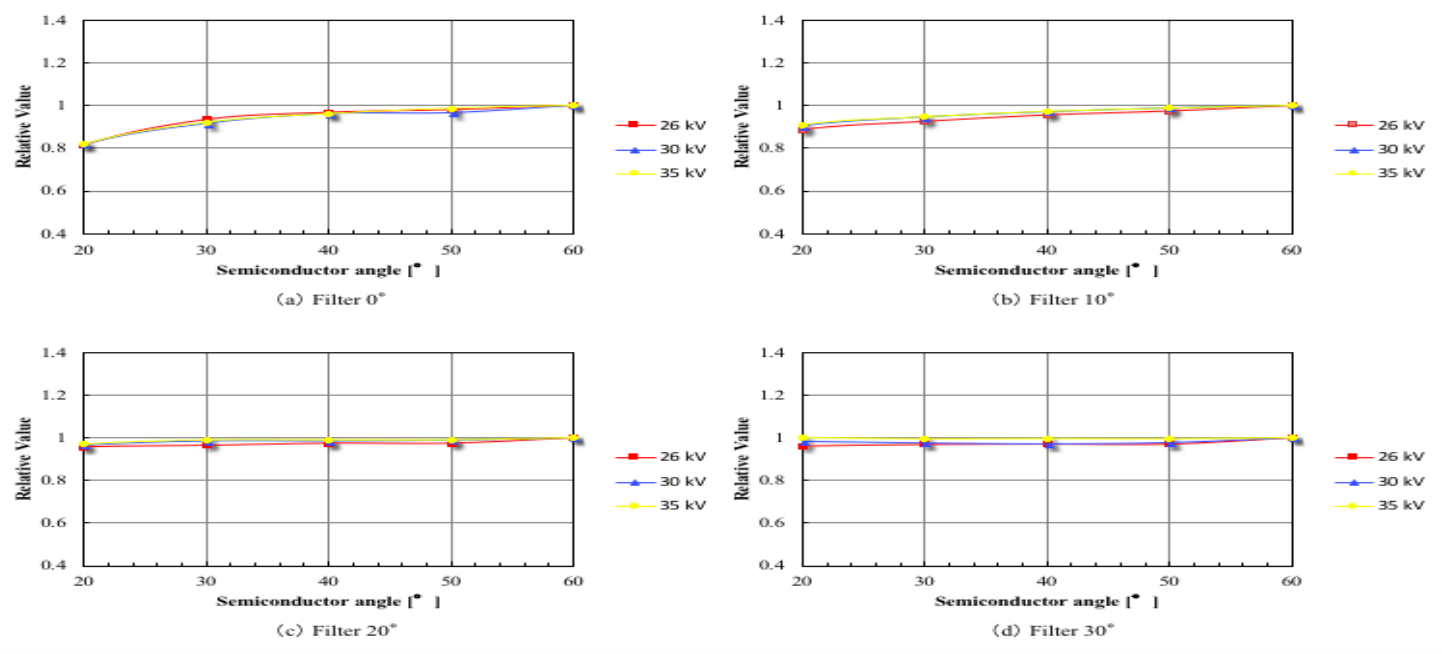

Fig 9:-Measurement results regarding angle characteristics of Mo filter and the detector of the dosimeter (Mo target)

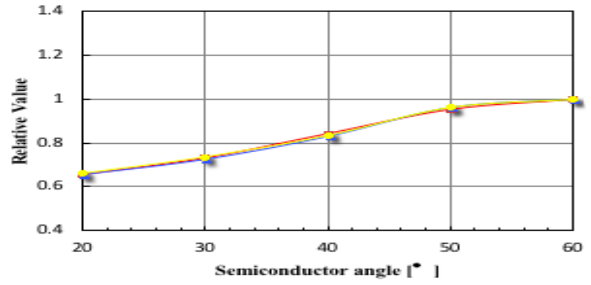

(a) Filter $0^{*}$

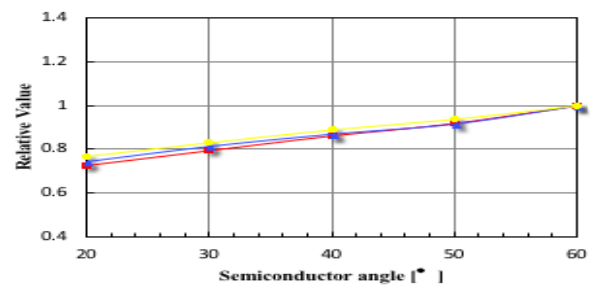

(c) Filter $20^{\circ}$

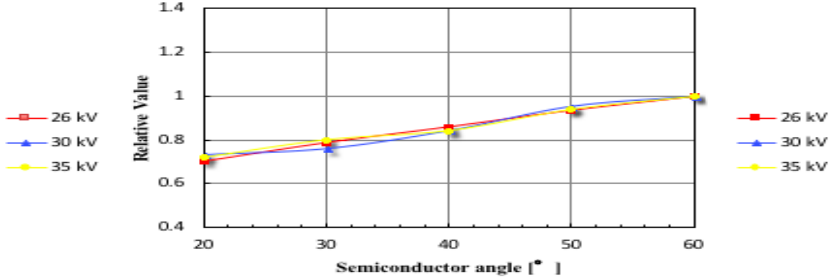

(b) Filter $10^{\circ}$

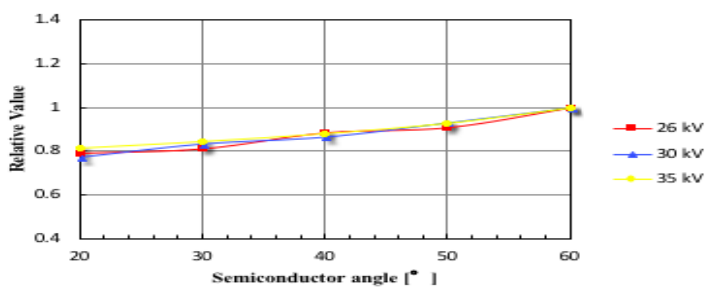

(d) Filter $30^{\circ}$

Fig 10:-Measurement results regarding angle characteristics of $\mathrm{Rh}$ filter and the detector of the dosimeter (Mo target) 


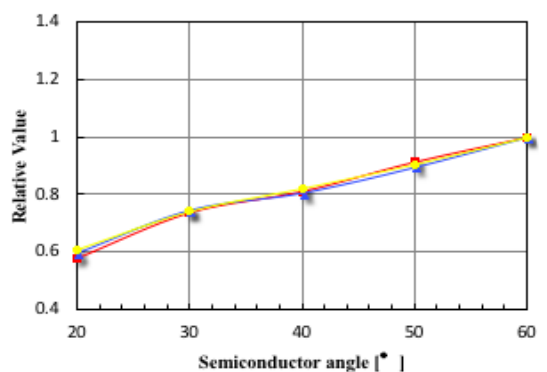

(a) Filter $0^{\circ}$

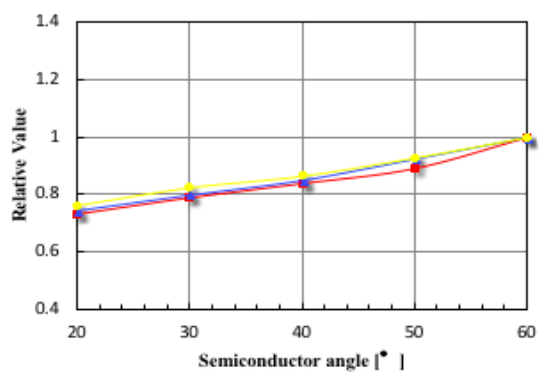

(c) Filter $20^{\circ}$
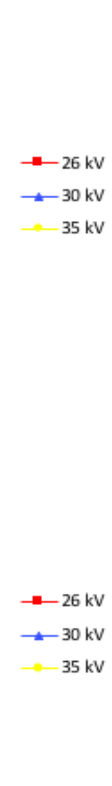

Fig 11:-Measurement results regarding angle characteristics of $\mathrm{Rh}$ filter and the detector of the dosimeter (W target)

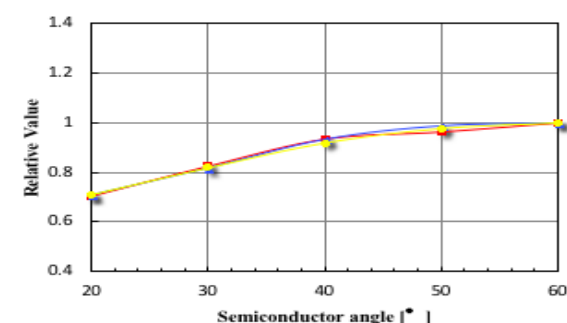

(a) Filter $0^{\circ}$

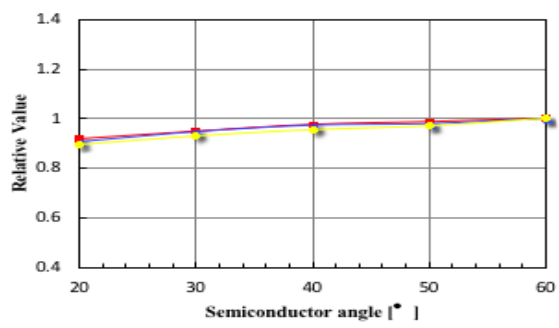

(c) Filter $20^{\circ}$

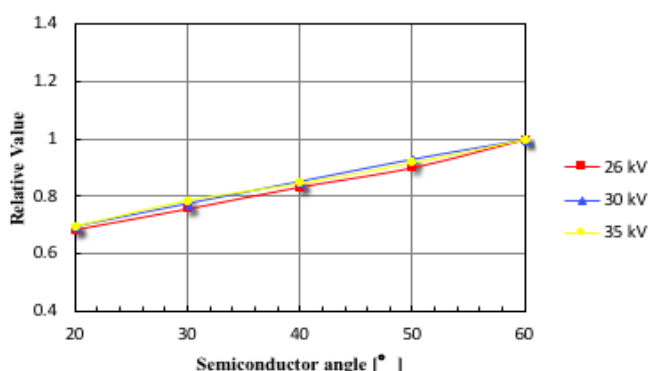

(b) Filter $10^{\circ}$

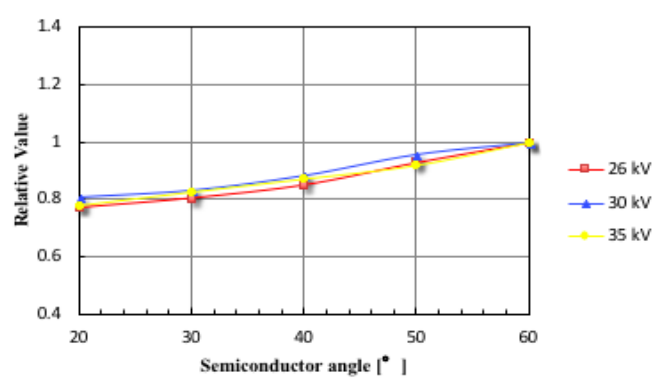

(d) Filter $30^{\circ}$
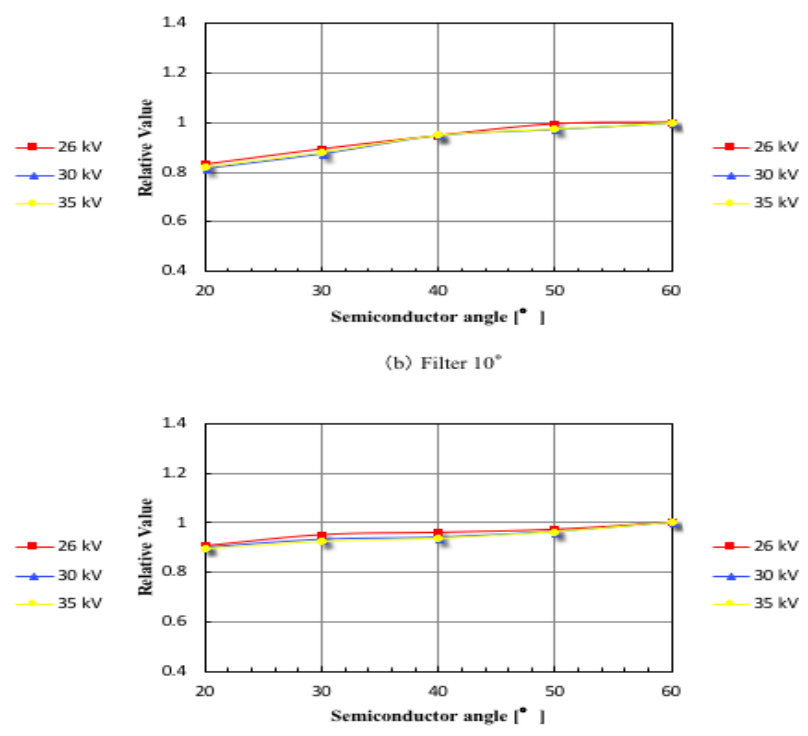

(d) Filter $30^{\circ}$

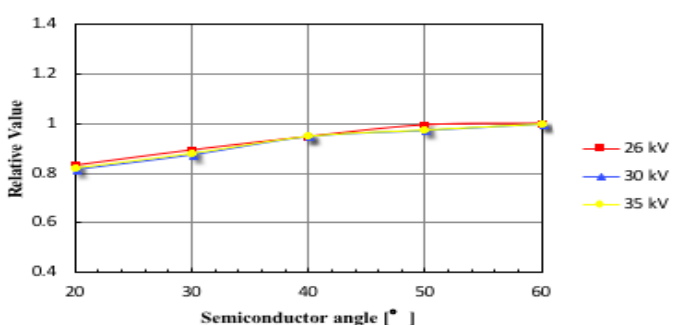

(b) Filter $10^{\circ}$

Fig 12:-Measurement results regarding angle characteristics of Ag filter and the detector of the dosimeter (W target) 

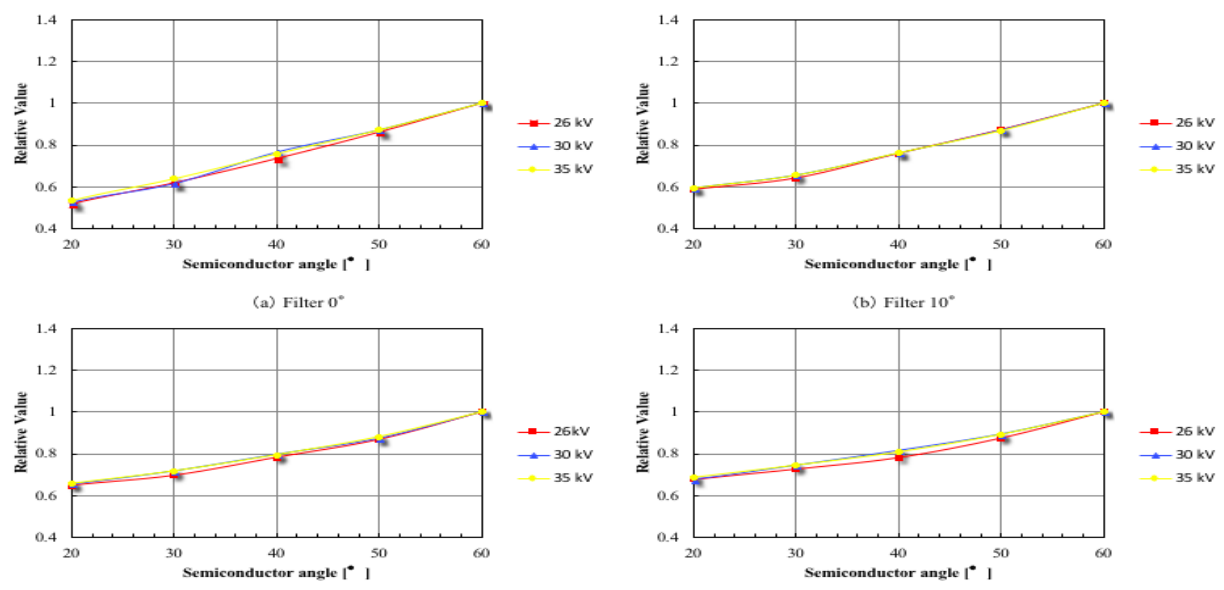

(c) Filter $20^{\circ}$

(d) Filter $30^{\circ}$

Fig 13:-Measurement results regarding angle characteristics of $\mathrm{Al}$ filter and the detector of the dosimeter (W target)

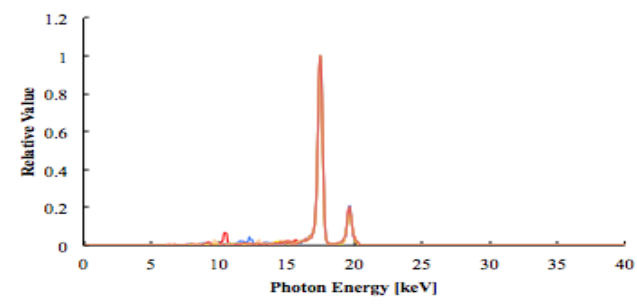

(a)

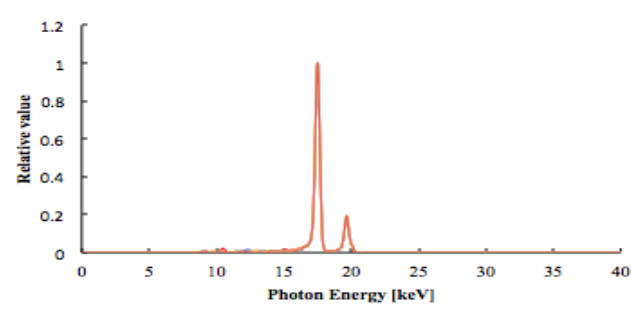

(c)

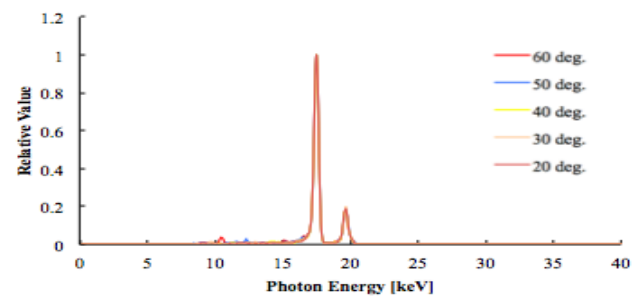

(b)

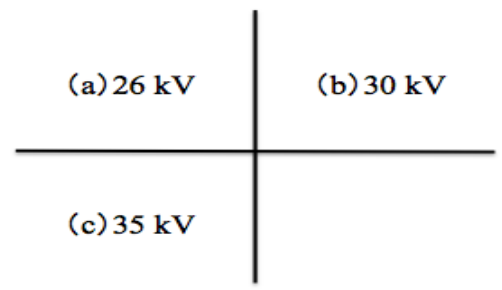

Fig 14:-Measurement results regarding spectrum of scattered radiations from Mo filter (Mo target)

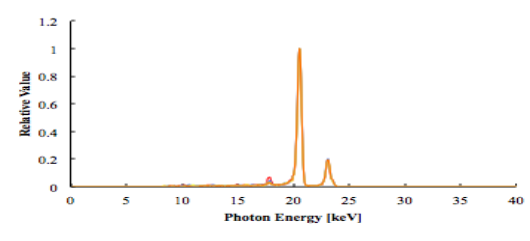

(a)

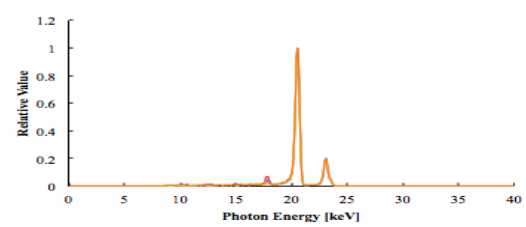

(c)

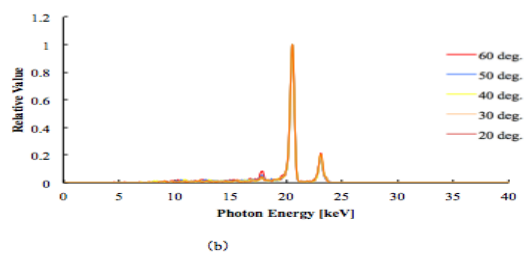

(b)

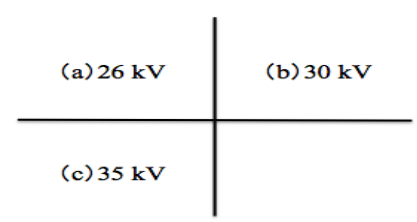

Fig 15:-Measurement results regarding spectrum of scattered radiations from Rh filter (Mo target) 


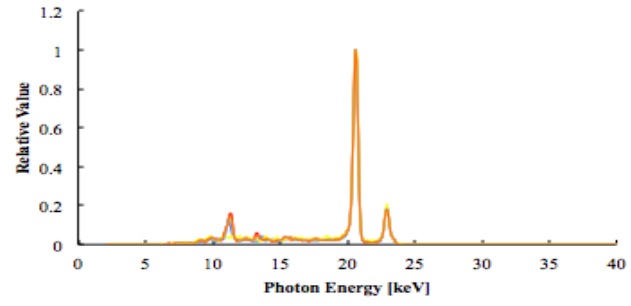

(a)

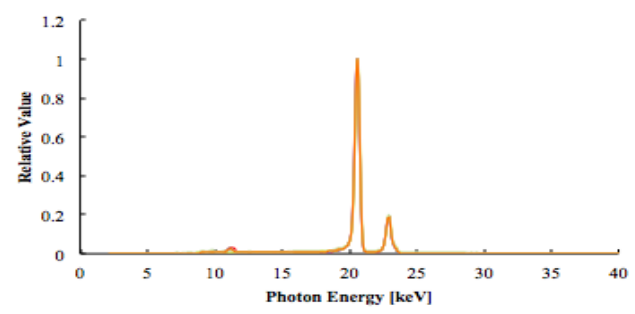

(c)

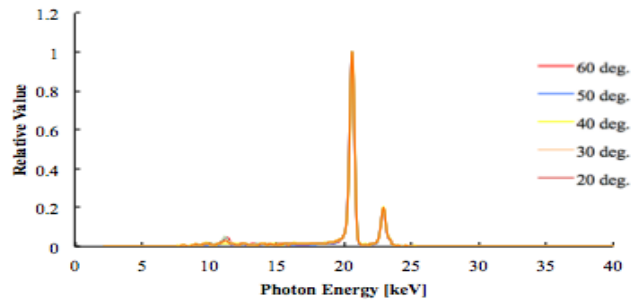

(b)

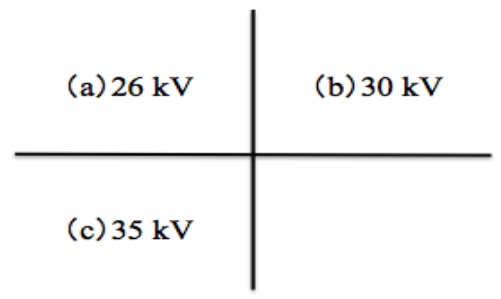

Fig 16:-Measurement results regarding spectrum of scattered radiations from $\mathrm{Rh}$ filter (W target)

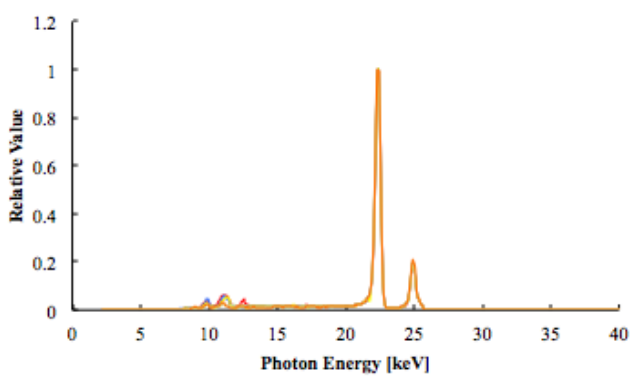

(a)

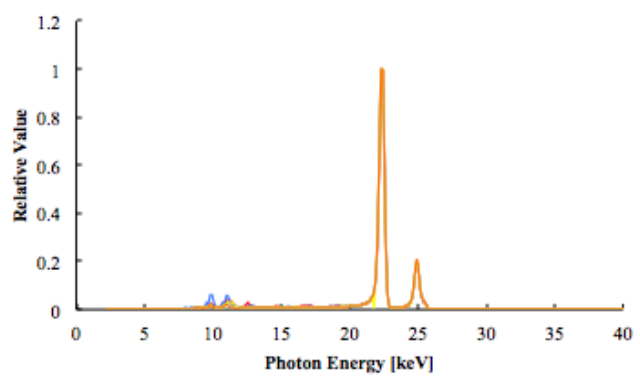

(c)

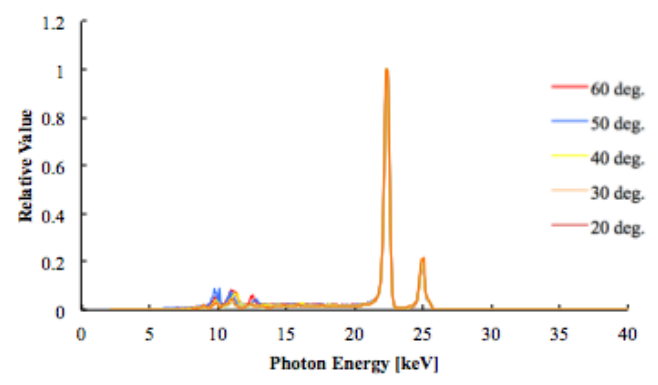

(b)

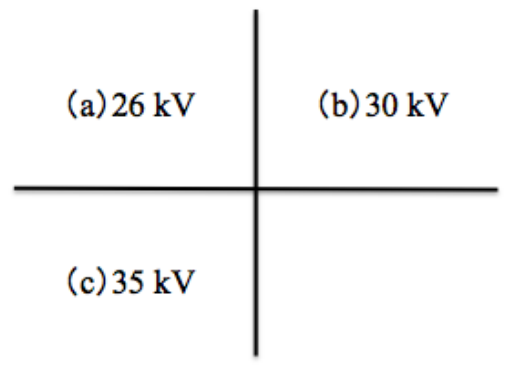

Fig 17:-Measurement results regarding spectrum of scattered radiations from Ag filter (W target) 


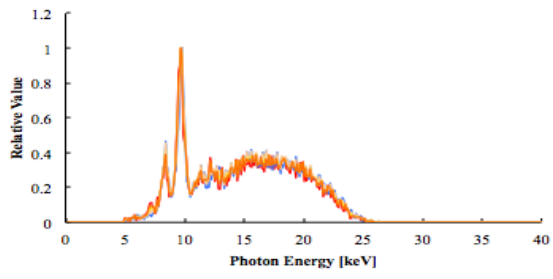

(a)

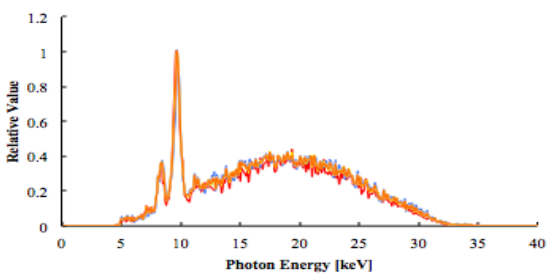

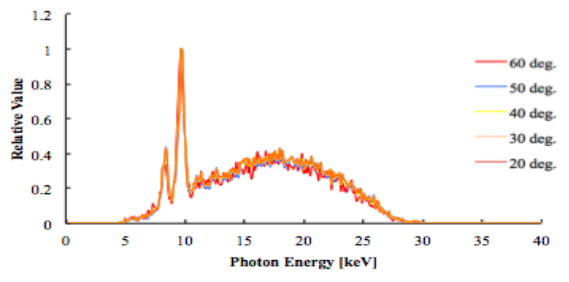

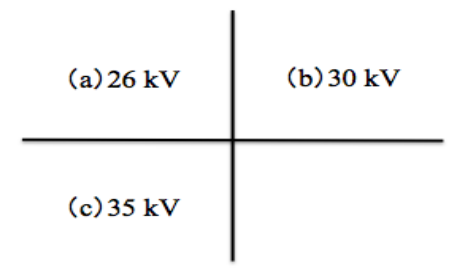

Fig 18:-Measurement results regarding spectrum of scattered radiations from $\mathrm{Al}$ filter (W target)

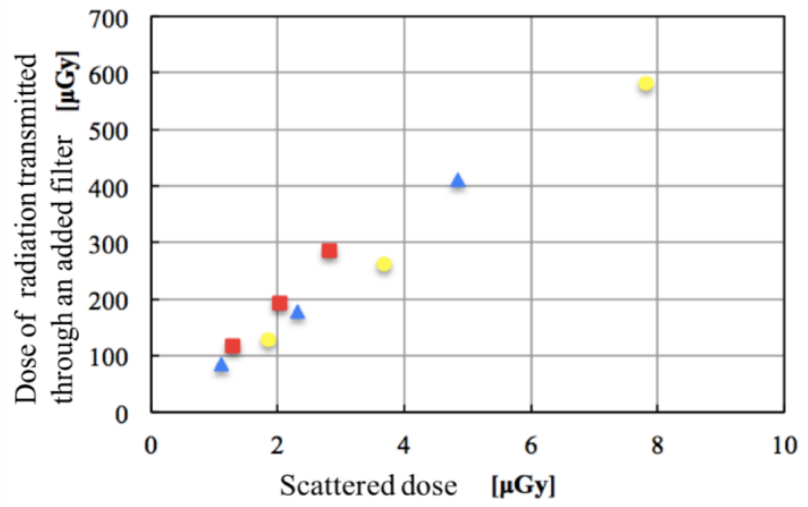

$26 \mathrm{kV} \mathrm{R}{ }^{2}=0.99755$

$\triangle 30 \mathrm{kV} \mathrm{R}{ }^{2}=0.99811$

$35 \mathrm{kV} \mathrm{R}{ }^{2}=0.99976$

Fig 19:-Measurement results regarding relationship between scattered radiation dose and dose of radiation transmitted through a Mo filter (Mo target)

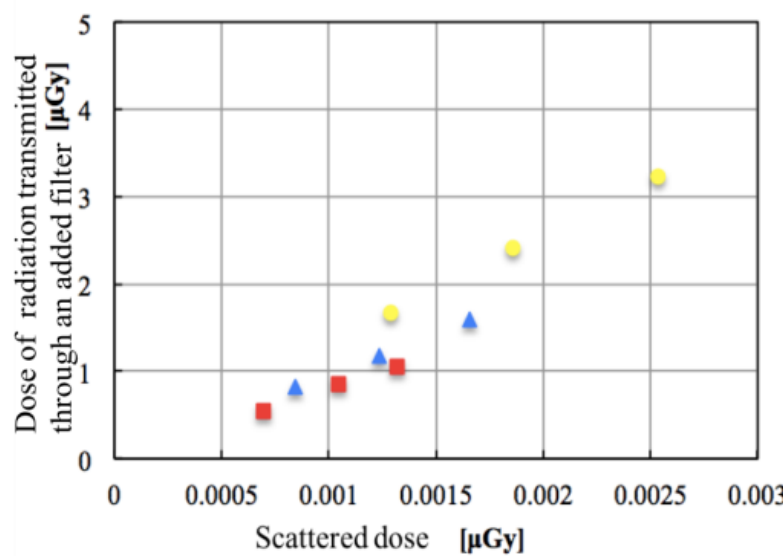

$26 \mathrm{kV} \mathrm{R}{ }^{2}=0.99849$

$\triangle 30 \mathrm{kV} \mathrm{R}^{2}=0.99872$

$35 \mathrm{kV} \mathrm{R}^{2}=0.99960$

Fig 20:-Measurement results regarding relationship between scattered radiation dose and dose of radiation transmitted through a $\mathrm{Rh}$ filter (Mo target) 


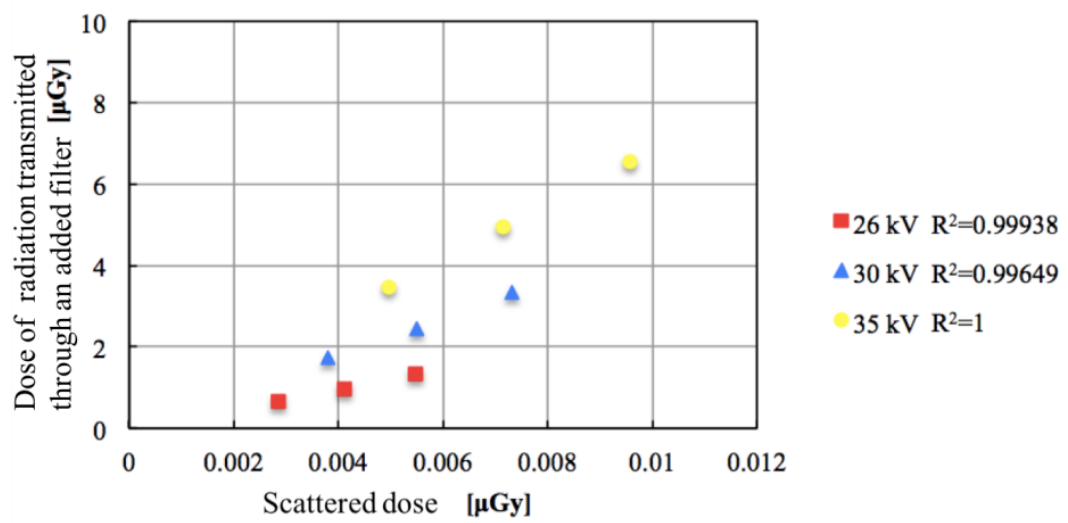

Fig 21:-Measurement results regarding relationship between scattered radiation dose and dose of radiation transmitted through a Rh filter (W target)
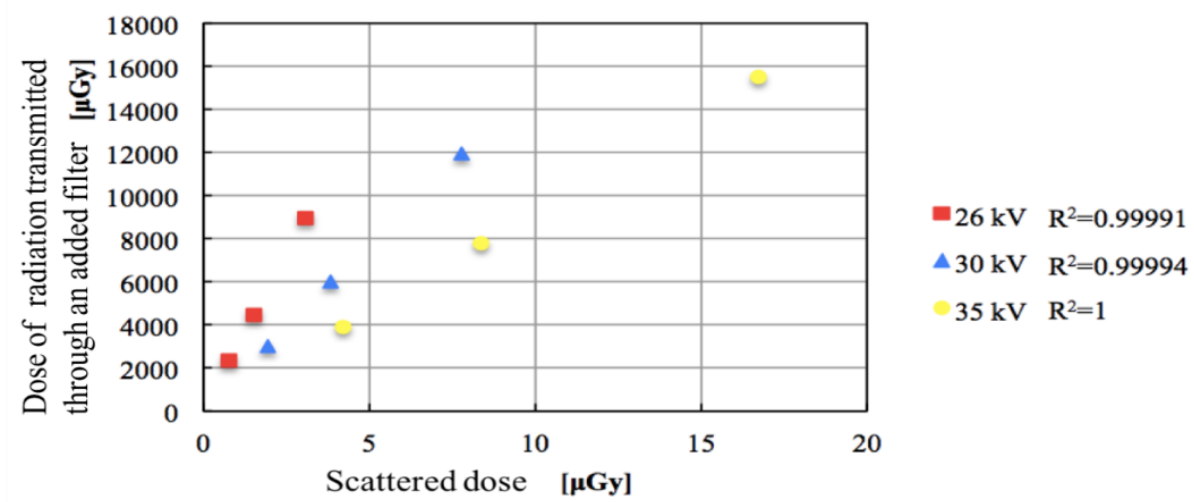

Fig 22:-Measurement results regarding relationship between scattered radiation dose and dose of radiation transmitted through a $\mathrm{Ag}$ filter (W target)

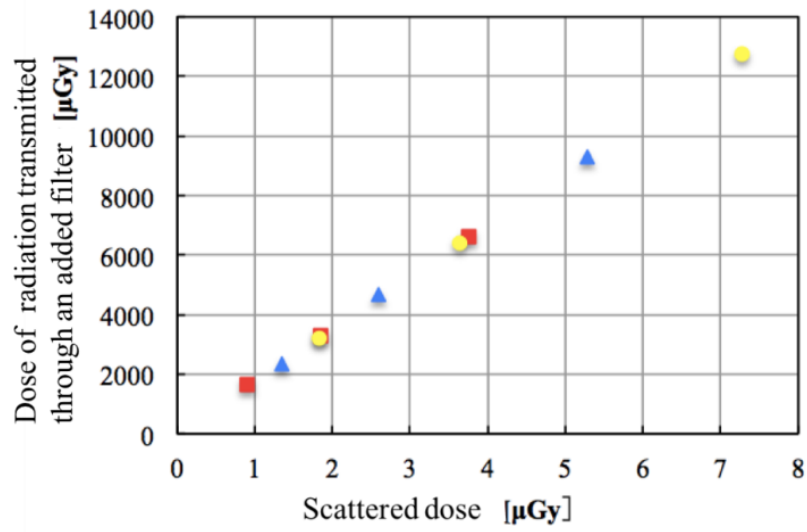

\footnotetext{
- $26 \mathrm{kV} \mathrm{R} \mathrm{R}^{2}=0.99999$

$\triangle 30 \mathrm{kV} \mathrm{R} \mathrm{R}^{2}=0.99967$

$35 \mathrm{kV} \mathrm{R} \mathrm{R}^{2}=1$
}

Fig 23:-Measurement results regarding relationship between scattered radiation dose and dose of radiation transmitted through a $\mathrm{Al}$ filter (W target) 
Table 1:-The measurement conditions of incidence direction characteristics

\begin{tabular}{|c|c|c|c|}
\hline target & tube voltage $[\mathrm{kV}]$ & tube current $[\mathrm{mA}]$ & irradiation duration [s] \\
\hline Mo & $26,28,35$ & 3 & 10 \\
\hline $\mathrm{W}$ & 30 & 1 & 10 \\
\hline
\end{tabular}

Table 2:-The measurement conditions of angle characteristics of added filters and the detector of the dosimeter

\begin{tabular}{|c|c|c|c|}
\hline target/added filter & tube voltage [kV] & tube current [mA] & irradiation duration [s] \\
\hline Mo/Mo & $26,30,35$ & 4 & 10 \\
\hline Mo/Rh & $26,30,35$ & 20 & 10 \\
\hline W/Rh & $26,30,35$ & 20 & 10 \\
\hline W/Ag & $26,30,35$ & 20 & 10 \\
\hline W/Al & $26,30,35$ & 4 & 10 \\
\hline
\end{tabular}

Table 3:-The measurement conditions of spectrum of scattered radiations from added filters

\begin{tabular}{|c|c|c|c|}
\hline target/added filter & tube voltage [kV] & tube current [mA] & irradiation duration [s] \\
\hline Mo/Mo & $26,30,35$ & 20 & 100 \\
\hline Mo/Rh & $26,30,35$ & 20 & 200 \\
\hline W/Rh & $26,30,35$ & 20 & 200 \\
\hline W/Ag & $26,30,35$ & 20 & 100 \\
\hline W/Al & $26,30,35$ & 20 & 300 \\
\hline
\end{tabular}

Table 4:-The measurement conditions of relationship between scattered radiation dose and dose of radiation transmitted through an added filter

\begin{tabular}{|c|c|c|c|}
\hline target/added filter & tube voltage [kV] & tube current [mA] & irradiation duration [s] \\
\hline Mo/Mo & $26,30,35$ & $5,10,15,20$ & 5,10 \\
\hline Mo/Rh & $26,30,35$ & $5,10,15,20$ & 5,10 \\
\hline W/Rh & $26,30,35$ & $5,10,15,20$ & 5,10 \\
\hline W/Ag & $26,30,35$ & $5,10,20$ & 5,10 \\
\hline W/Al & $26,30,35$ & $5,10,20$ & 5,10 \\
\hline
\end{tabular}

\section{Conclusions:-}

This study aimed to basically examine a method of measuring actual AGD of individual examinees in mammography, and assessed use of a semiconductor dosimeter for measurement of an X-ray output without changing the radiation quality. The measurement conditions, that are likely optimal judging from the examination results, are as follows:

1. The semiconductor dosimeter is to be cooled constantly due to its temperature characteristics.

2. The straight line connecting the added filter center and the semiconductor dosimeter's detector center is to be orthogonal to the detection plane of the dosimeter's detector constantly.

3. The dosimeter's detector is to be opposed, at an angle not blocking the primary X-ray, to the added filter as much as possible.

4. It is likely possible to calculate the X-ray output in mammographic X-ray apparatuses by multiplying the dose of the scattered radiation measured in the layout above by the respective calibration constant of each target/added filter combination. In addition, we believe that it is possible to grasp AGD by examinee by multiplying the X-ray output by coefficients additionally. 


\section{Future perspective:-}

This study used an industrial X-ray apparatus, causing measurements in conditions in which size of added filters, irradiation field, measurement distance, etc. were different from those of actual mammographic X-ray apparatuses. Therefore, it is necessary in future to make measurement conditions closer to the actual conditions to examine the calibration constant for AGD calculation. Moreover, an additional experiment on a cooling method of the semiconductor dosimeter by use of a Peltier element is necessary.

Additionally, regarding DBT, which began being prevalent recently, oblique X-ray incidence to the dosimeter becomes a problem in measurements because the system rotates an X-ray tube in a fan-like manner. Although a shallow ionizing chamber is usually used in the mammography field, the shallow type is not suitable for oblique Xray incidence in DBT because of its directional dependence in terms of structure. Therefore, AGD is calculated through vertical X-ray irradiation to the reference plane of the dosimeter and correction by multiplication by various coefficients. However, based on this study, we believe that it may be possible to use a semiconductor dosimeter, in other words, coefficients for correcting oblique X-ray incidence would be unnecessary in AGD calculation, enabling simple AGD calculation. Hence, it is necessary to examine a correlation between measurement values by use of oblique X-ray incidence and those by scattered radiation in DBT systems.

\section{Reference:-}

1. Setting of diagnostic reference level based on latest domestic actual survey result (2015). [online]Available at: http://www.radher.jp/JRIME/report/DRLhoukokusyo.pdf\#search=\%27\%E6\%9C\%80\%E6\%96\%B0\%E3\%81\% AE\%E5\%9B\%BD\%E5\%86\%85\%E5\%AE\%9F\%E6\%85\%8B\%E8\%AA\%BF\%E6\%9F\%BB\%E7\%B5\%90\%E6 \%9E\%9C\%Е3\%81\%AB\%E5\%9F\%BA\%E3\%81\%A4\%E3\%82\%99\%Е3\%81\%8F\%E8\%A8\%BA\%Е6\%96\%A D\%E5\%8F\% 82\%E8\%80\%83\%Е3\%83\%AC\%Е3\%83\%98\%Е3\%82\%99\%Е3\%83\%AB\%Е3\%81\%AE\%Е8\%A 8\%AD\%E5\%AE\%9A\%27 [Accessed 7 July. 2018].

2. The Japan Central Organization on Quality Assurance of Breast Cancer Screening. Digital Mammography Quality Control Manual 2009: 19-25.

3. Japanese society of radiological technology. Mammographic quality control manual 2015; 14-4: 12, 35-50.

4. Otsuka E, Hiyamizu S, Ohyama T. Latest semiconductor engineering 1993: 27-30.

5. Sekimoto M, Katoh Y, Katoh T. Calibration coefficients of dosimeters used in mammography for various target/filter combinations. J Appl Clin Med Phys 2015 Nov; 16(6): 401-410.

6. Yamada K, Nohara H. Radiation metrology 1981: 211-212.

7. Saegusa K, Irifune T, Fukushi M. Radiation basic metrology 2011: 145-147.

8. European Reference Organization for Quality Assured Breast Screening and Diagnostic Services. Protocol for the Quality Control of the Physical and Technical Aspects of Digital Breast Tomosynthesis Systems version 1.0 2013. 